\title{
Design and Implementation of a Scalable General High Performance Remote Sensing Satellite Ground Processing System on Performance and Function
}

\author{
Jingshan Li and Dingsheng Liu \\ Center for Earth Observation and Digital Earth, Chinese Academy of Sciences, \\ No.45, Bei San Huan Xi Road, Beijing, China \\ \{jsli,dsliu\}@ceode.ac.cn
}

\begin{abstract}
This paper discusses design and implementation of a scalable high performance remote sensing satellite ground processing system using a variety of advanced hardware and software application technology on performance and function. These advanced technologies include the network, parallel file system, parallel programming, job schedule, workflow management, design patterns,etc, which make performance and function of remote sensing satellite ground processing system scalable enough to fully meet the high performance processing requirement of multi-satellite, multi-tasking, massive remote sensing satellite data. The "beijing-1" satellite remote sensing ground processing system is introduced as an instance.
\end{abstract}

Keywords: parallel computing, remote sensing, data processing system, clusters computing, scalable.

\section{Introduction}

With development of the remote sensing mini-satellites, mini-satellites constellation, as well as the high-performance parallel computing technology, the remote sensing satellite ground processing system faces to the demand of multi-platform, multitasking, high performance data processing. Remote sensing is characterized by a need to perform computationally intensive operations on large data sets. Full-scene image processing requires operating on tens of millions of image data points per scene.

The cluster of PC server has grown from a curiosity to become the norm for much of the world's computing [1],[5],[6].Integrating parallel computer programs into a framework that can be easily used by applied scientists is a challenging problem. Such a framework has to enable simplified access to computationally complex operations and high performance technologies, as well as providing a means for defining the appropriate data sets for the operation request. It still can be inadequate for a lack of suitable software, suitable hardware and total integrate processing system [2], [3],[4].

The scalable General HIgh Performance remote sensing satellite ground Processing System (GHIPS) is researched. The advanced high performance computing technology, advanced software design, a combination of parallel storage technologies, parallel computing, task scheduling technology, process management technology, design 
methods, web service technology are used in this system, which makes performance and function to achieve a high degree to meet the requirement of satellite ground data processing system.

We describe our GHIPS design on performance and function in section 2 and GHIPS implementation case, the "beijing-1" satellite remote sensing ground processing system, in section 3. We discuss conclusion in section 4.

\section{GHIPS Design on Performance and Function}

GHIPS, developed at the Center for Earth Observation and Digital Earth, Chinese Academy of Sciences, is a high performance computing middleware that supports the development and execution of generic remote sensing satellite ground processing system applications over a collection of hardware and software resources. The system is client-server architecture. The client submitted to the task of processing through the web service protocol to server end, and server end finished product processing with high performance.

This paper focuses on the server-side design of the system. The key issue of remote sensing satellite ground processing system on performance and function is researched.

As shown in Figure 1, the server end system is divided into five layers, namely the hardware layer, system support layer, data processing layer, tasks scheduling layer and user composition layer. The first two layers make system scalable on performance and the other three layers make system scalable on function. The methods of making system scalable on performance and function are discussed below.

\begin{tabular}{|c|c|c|}
\hline \multicolumn{3}{|c|}{ User Defined Workflow } \\
\hline \multicolumn{3}{|c|}{ Workflow Management } \\
\hline \multicolumn{3}{|c|}{ Job Schedule } \\
\hline \multicolumn{3}{|c|}{ Abstract Processing Function Interface } \\
\hline \multicolumn{3}{|c|}{ Concrete Processing Function Implementation } \\
\hline Parallel Computing & Parallel Storage & $\begin{array}{c}\text { Network Load } \\
\text { Balance }\end{array}$ \\
\hline
\end{tabular}

User Composition Layer

Task Scheduling Layer

Data Processing Layer

System Support Layer

Hardware Layer

Fig. 1. GHIPS Server End Architecture

\subsection{Hardware Layer}

The hardware layer provides hardware support, including commercial off-the-shelf systems such as PC server cluster, Storage Area Network (SAN) / Network Attached Storage (NAS) storage systems, Gigabit Ethernet or Infiniband network systems.

All three modes use traditional network storage technologies: direct attached storage (DAS), which is attached to a single server; NAS, a server dedicated to file sharing; and SAN, networks of shared storage devices. NAS can offer low cost massive 
storage, and SAN can offers both high performance network storage and secure data sharing across platforms.

The InfiniBand is a switched fabric communications link primarily used in highperformance computing. Its features include quality of service and failover, and it is designed to be scalable. The InfiniBand architecture specification defines a connection between processor nodes and high performance I/O nodes such as storage devices. The InfiniBand can offer high-bandwidth, low-latency communication among cluster. The InfiniBand standard supports single data rate (SDR), double data rate (DDR), and quad data rate (QDR), which provides bandwidth equal to 10Gbps, 20Gbps, and 40Gbps, respectively.

The commercial off-the-shelf computing hardware is a cost-effective way of exploiting in remote sensing applications. The above high performance hardwares have standard interface and make system scalable on performance of computing, storage and network. On this hardware layer, users can deployment commercial off-the-shelf software without worrying about any further change of hardware.

\subsection{System Support Layer}

The remote sensing data processing is a complex computing intensive, data intensive and network intensive applications and different functions of computing, storage and network access performance requirements may differ from each other.

The system support layer provides software support environment, includes parallel storage software, such as Stornext, PVFS, Luster file system, parallel computing software, such as MPI, OpenMP, PVM, network load balance software, such as LVS, BigIP. This layer can integrate and make full use of computing, storage, and network access software and resource. The orthogonal design of the system of parallel computing, parallel storage, the parallel network load make the system performance, network performance, storage performance to be scalable independent, and to be optimized data processing performance on the three dimensions. The optimized method of three dimensions is showed in Figure 2.This layer shields the complexity of using of parallel software, and users can design and implement their data processing application without worrying about any further change of parallel system support software, and can gain the better performance of new system software and hardware automatically.

\subsection{Data Processing Layer}

The data processing layer makes use of advanced software design patterns. In software engineering, a design pattern is a general reusable solution to a commonly occurring problem in software design. A design pattern is not a finished design that can be transformed directly into code. It is a description or template for how to solve a problem that can be used in many different situations. Object-oriented design patterns typically show relationships and interactions between classes or objects, without specifying the final application classes or objects that are involved. 


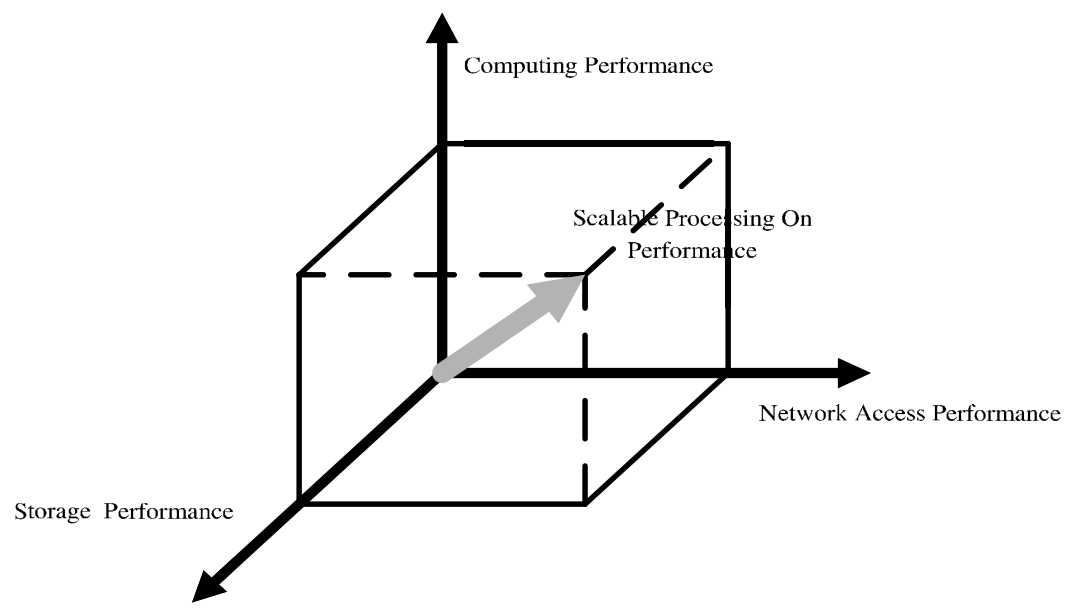

Fig. 2. Scalable on Performance

A software design pattern, the abstract factory Pattern provides a way to encapsulate a group of individual factories that have a common theme. In normal usage, the client software would create a concrete implementation of the abstract factory and then use the generic interfaces to create the concrete objects that are part of the theme. The client does not know (or care) about which concrete objects it gets from each of these internal factories since it uses only the generic interfaces of their products. This pattern separates the details of implementation of a set of objects from its general usage.

The template design pattern is perhaps one of the most widely used and useful design patterns. It is used to set up the outline or skeleton of an algorithm, leaving the details to specific implementations later. This way, subclasses can override parts of the algorithm without changing its overall structure. This is particularly useful for separating the variant and the invariant behavior, minimizing the amount of code to be written. The invariant behavior is placed in the abstract class (template) and then any subclasses that inherit it can override the abstract methods and implement the specifics needed in that context.

Using design pattern, data processing layer design a variety of abstract function interface for a variety of satellite sensors using abstract factory design pattern and common logical thread of public functions using template method design pattern. The various processing algorithms of satellite sensors can be added through inherit, which ensure scalability of satellite sensors data processing algorithms. The scalable data processing layer architecture showed as class diagram is illustrated in Figure 3, for examples of data processing of "beijing-1" satellite and " CBERS " satellite.

\subsection{Task Scheduling Layer}

A cluster system has driven the need for new job scheduling system in the computational science realm. A job scheduling system is used for both serial and parallel job control. 


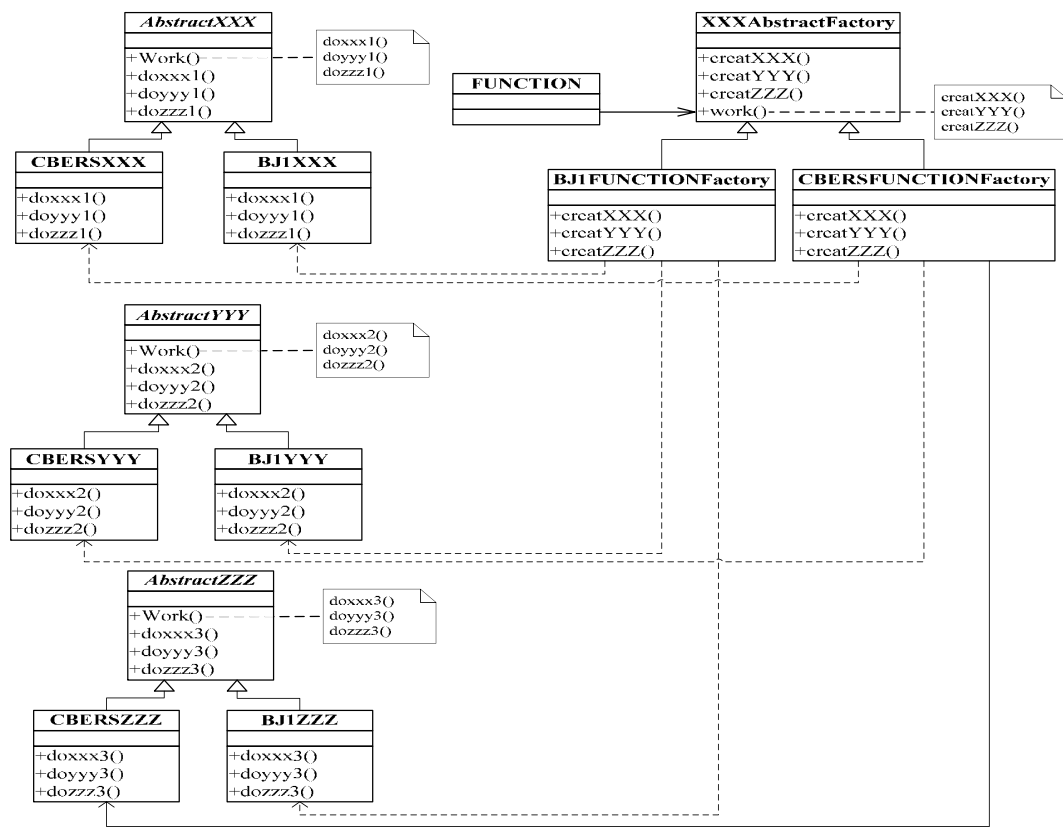

Fig. 3. Scalable Data Processing Layer Architecture and Class Diagram

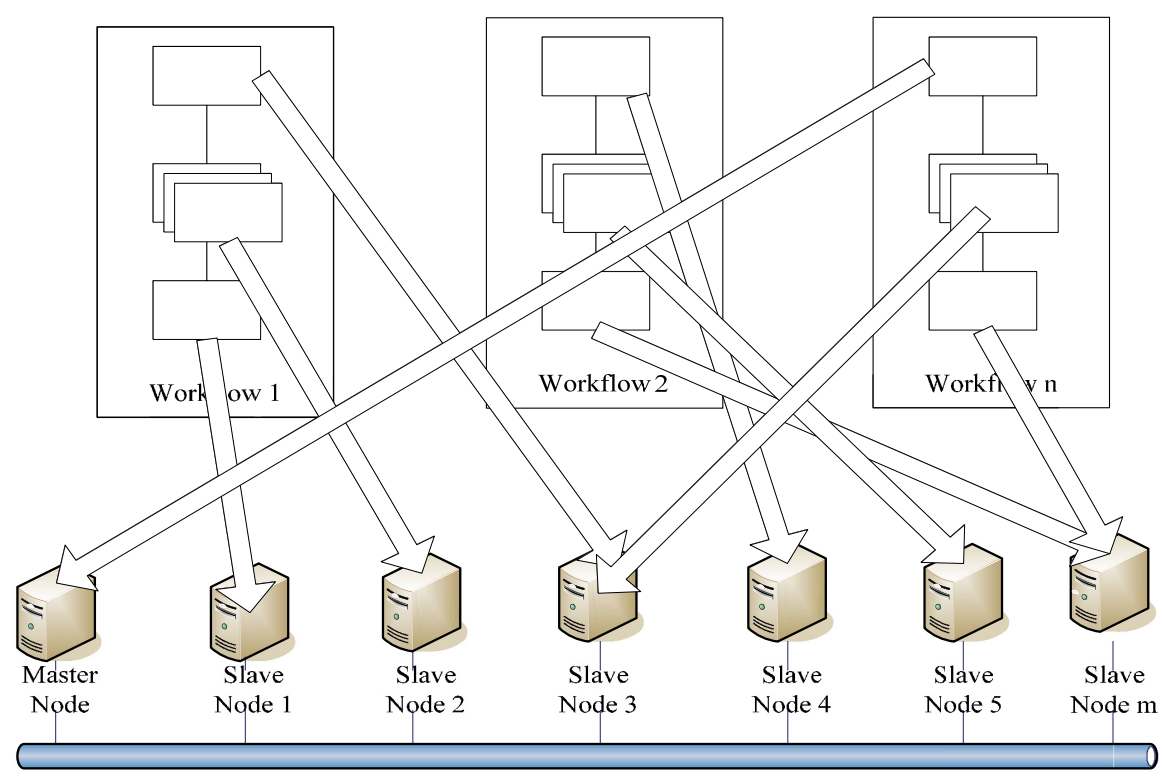

Fig. 4. Tasks Scheduling System 
A workflow is a depiction of a sequence of operations, declared as work of a person, work of a simple or complex mechanism, work of a group of persons, work of an organization of staff, or machines. Workflow software can provide end users with an easier way to orchestrate or describe complex processing.

The tasks scheduling layer integrate job scheduling and workflow management system to achieve the work load balancing process and the ability to compose complex job workflow. The tasks scheduling layer can assign any data processing job on the whole cluster with help of system support software. The tasks schedule is illustrated in Figure 4.

\subsection{User Composition Layer}

The user composition layer provides users with kind interface to compose process, users can compose a variety of complex processing, such as data processing, archiving, production, distribution and other functions, as shown in Figure 5.

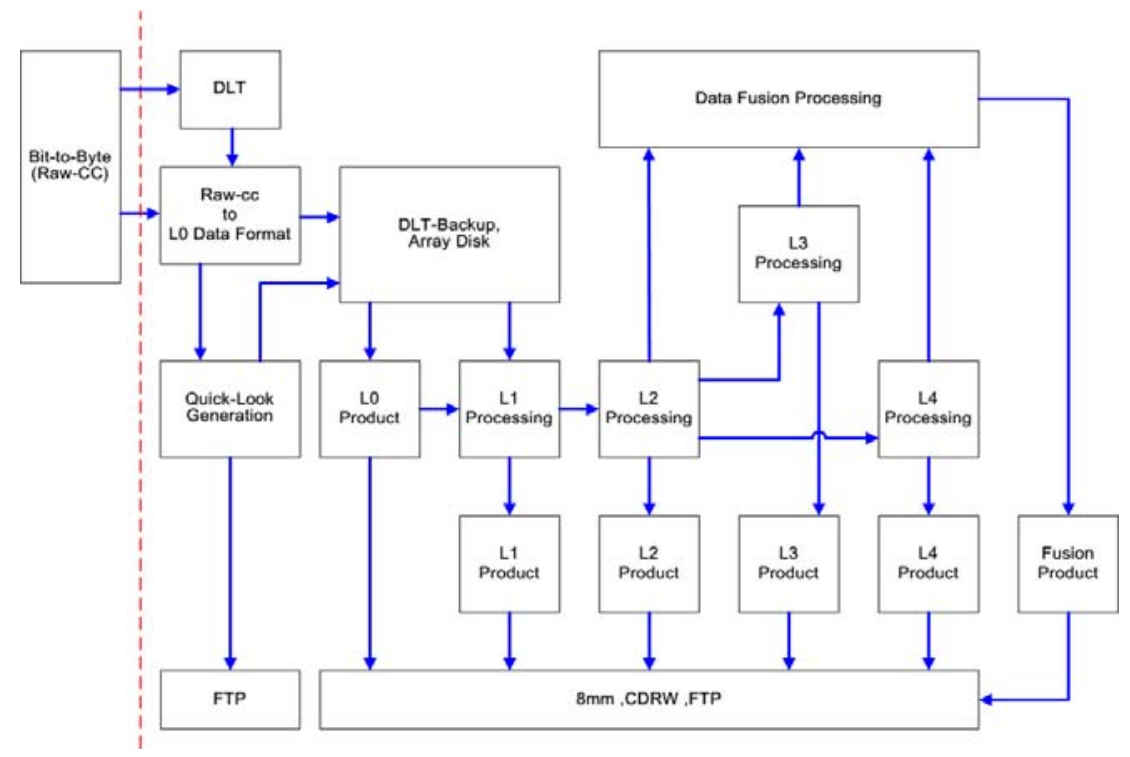

Fig. 5. User Composition Data Processing Task

\subsection{Scalable on Performance and Function}

The hardware layer and system support layer achieve on demand scalability on system performance. The using of high performance commercial off-the-shelf computing hardware and orthogonal design of the system of parallel computing, parallel storage, and the parallel network load make the system performance, network performance, storage performance to be scalable independent, and to be optimized data processing performance on the three dimensions.

The data processing layer, task scheduling layer and user composition layer achieve on demand scalability on system function, that not only to increase the number of new satellite remote sensing data processing algorithms and functions, but also 
bring out a variety of composition of the basic processing functions. Users can customize remote sensing data processing to meet user custom needs of data processing.

The system has a completely open platform due to the use of a variety of commercial off-the-shelf advanced high performance computing technology and advanced software design technology. Users can facilitate the balanced expansion of the system of processing, storage and network capacity to improve the performance of the process and load their own business functions of a new satellite remote sensing data by inserting the appropriate data processing module based on certain rules to extend system processing function without modifying the system hardware and software.

\section{GHIPS Implementation Case}

Based on above research, The new version of "Beijing-1" remote sensing satellite ground processing system is implemented on GHIPS, which make use of NAS storage systems, Gigabit Ethernet, and computing servers hardwares, PVFS2,Parallel Virtual File System Version 2,MPICH2,an implementation of the Message-Passing Interface, openPBS, open source version of the Portable Batch System, OSworkflow, a pure Java open-source workflow engine for technical users, and JAVA RMI, Remote Method Invocation.

The system include system geometric correction, geometric correction based on control point and Digital Elevation Model (DEM), and other functions more than a dozen modules of serial and parallel processing, more than $30 \mathrm{kinds}$ of business of composition of modules, and more business can be composed in accordance with the requirements of users.

The cluster consists of 16 SMP PC computing nodes connected by 24 port gigabit Ethernet switch. The computing node has two Intel Xeon 2.4GHz CPU and $1 \mathrm{G}$ Bytes local memory. The eight nodes are used for new version based on GHIPS; the other eight nodes are used for previous version of "Beijing-1" remote sensing satellite ground processing system.

The test data processing includes raw data management, band registration, MTF correction. The system shows about $20 \%$ performance improvement over the previous version with using the local file system and custom scheduling system on the same hardware. The test data and result are showed in table 1 .

Table 1. New version based on GHIPS versus previous version

\begin{tabular}{||c|c|c|c||}
\hline \multirow{2}{*}{ Dataset Name } & Data Row Number & \multicolumn{2}{|c||}{ time-consuming(second) } \\
\cline { 3 - 4 } & & New version & Previous version \\
\hline $\begin{array}{c}\text { dmc+4bj1L104081701 } \\
\text { 60041_160155_0 }\end{array}$ & $5000 \sim 14983$ & 777 & 851 \\
\hline $\begin{array}{c}\text { dmc+4bj1L104081880 } \\
\text { 32203_032703_0 }\end{array}$ & $10000 \sim 19983$ & 711 & 896 \\
\hline $\begin{array}{c}\text { dmc+4bj1L104081880 } \\
\text { 32203_032703_0 }\end{array}$ & $50000 ~ 59983$ & 718 & 931 \\
\hline $\begin{array}{c}\text { dmc+4bj1L104081910 } \\
\text { 20724_020844_0 }\end{array}$ & 1 1 9984 & 654 & 903 \\
\hline
\end{tabular}




\section{Conclusions}

As many remote sensing satellite ground processing applications for computing have high demand for processing powers, it is critical to have a platform that readily supply such computing powers with the ease of use.

In this paper we propose the architecture of GHIPS. With using of a variety of commercial off-the-shelf advanced high performance computing technology and advanced software design technology, users can facilitate the balanced expansion of the system of processing, storage and network capacity to improve the performance of the process and load their own business functions of a new satellite remote sensing data by inserting the appropriate data processing module based on certain rules to extend system processing function without modifying the system hardware and software.

Future work is divided between application issues and infrastructure issues. The application issues include the integration new satellite remote sensing data processing and basic algorithms. The infrastructure issues are studying how parallelization at various hardware and software levels can be used for maximization of performance and researching corresponding algorithms parallel model.

\section{References}

1. Chao-Tung, Y., Chih-Li, C., Chi-Chu, H., Frank, W.: Using a BEOWULF cluster for a remote sensing application. In: Proceedings of the 22nd Asian Conference on Remote Sensing, Singapore, pp. 233-238 (2001)

2. Li, G., Liu, D.: Key Technologies Research on Building a Cluster-Based Parallel Computing System for Remote Sensing. In: International Conference on Computational Science, vol. (3), pp. 484-491 (2005)

3. Zhang, W., Liu, D., Li, G., Zhang, W.: Special Task Scheduling and Control of Cluster Parallel Computing for High-Performance Ground Processing System. In: Alexandrov, V.N., van Albada, G.D., Sloot, P.M.A., Dongarra, J. (eds.) ICCS 2006. LNCS, vol. 3993, pp. 17 23. Springer, Heidelberg (2006)

4. Hawick, K.A., Maciunas, K.J., Vaughan, F.A.: DISCWorld: A Distributed Information Systems Control System for High Performance Computing Resources. DHPC Technical Report DHPC-014, Department of Computer Science, University of Adelaide (1997)

5. Yang, C.T.: Using a Beowulf Cluster for a Remote Sensing Application. In: Proceedings of the Asian Conference on Remote Sensing (ACRS), Singapore (2001)

6. Sterling, T.L., Salmon, J., Backer, D.J., Savarese, D.F.: How to Build a Beowulf: A Guide to the Implementation and Application of PC Clusters 2nd Printing. MIT Press, Cambridge (1999) 